\title{
De nouvelles anomalies moléculaires dans des maladies de la choroïde et de la rétine
}

L'analyse moléculaire des maladies de la vision progresse rapidement. On connaît au moins deux gèn's's dont l'altération est responsable de rétinites pigmentaires, ceux de la rhod(opsine et de la périphérine $\left(m / s n^{\circ} 1 \mathrm{vol}\right.$. 8, p. 82 et $n^{\circ} 2$, vol. 8, p.171). C'est au tour des lésions choroïdiennes de focaliser l'attention. Une forme fréquente de cécité est liée au sexe, la choroïdérémie. Son gène a pu être localisé en Xq2.1. En 1990, aux Pays-Bas, Cremers et al. [1] ont isolé des clones d'ADNc codant pour une protéine de 316 acides aminés. Ils ont appelé ce gène hCHM (human choroideremia). L'ADN génomique s'est montré partiellement ou totalement absent par suite de délétion chez huit malades et, chez une femme atteinte, une translocation impliquait la région $\mathrm{Xq2.1}$. On trouve normalement l'ARN messager dans la choroïde, la rétine, mais aussi, bien qu'en quantité moindre, dans les lymphocytes immortalisés par le virus EBV. Ce messager est absent ou tronqué chez les sujets porteurs de délétions. Très récemment [2] le même groupe a identifié plusieurs mutations ponctuelles chez cinq malades, qui toutes provoquent l'apparition d'un codon de terminaison, entraînant la synthèse d'une protéine tronquée. Cette équipe de Cremers [3] vient d'effectuer une autre découverte importante. Partant d'un ADNc complet de souris, elle a identifié un gène très semblable à celui de la choroïdérémie, qu'elle a dénommé hCHML (human choroïderemia like). La protéine dont il dirige la synthèse est plus longue (656 acides aminés) mais un de ses domaines lui est analogue à $75 \%$. Ce nouveau gène diffère de celui que porte l'X surtout par deux points : sa localisation est autosomique, en 1q3.1-qter; surtout sa structure est différente : alors que le gène porté par l'X s'étend sur environ $60 \mathrm{~kb}$ et contient au moins sept introns [1], le gène autosomique ne couvre que un peu plus de $2 \mathrm{~kb}$ et légitime de penser que $h C H M L$ dérive d'ARN de $h C H M$, par transcription inverse et intégration dans le génome. On sait qu'un tel phénomène n'est pas exceptionnel pour des gènes liés à l'X ; il a été décrit pour la phosphoglycérate kinase et une pyruvate déshydrogénase ; il permettrait de circonvenir l'inactivation de l'X ou son absence dans les cellules haploïdes masculines. S'il en est bien ainsi, le gène $h C H M$ devrait jouer un rôle indispensable lors de certaines étapes du développement. Les premières recherches sur ces protéines n'avaient montré aucun ressemblance entre elles et toute autre protéine connue. En 1991 Foder et al. (San Francisco, CA, USA) ont remarqué [4] qu'une homologie existait entre hCHM et une protéine appelée p25 AGDI, qui inhibe in vitro la libération du GDP d'une protéine de type ras. Cette p25 A-GDI pourrait avoir une relation avec la transducine, une protéine $G$ trimérique qui transmet les signaux dans la rétine. On peut rappeler à ce sujet que la dégénérescence rétinienne de la souris $r d$ est due à une anomalie de la cGMP phosphodiestérase des bâtonnets [5].

Le dernier développement, et non le moindre, a consisté en un rapprochement entre le gène de hCHML et d'un type de syndrome de Usher. Ce syndrome associe une surdité congénitale, une perte progressive de la vision par rétinite pigmentaire et des anomalies vestibulaires. On distingue un type I, dans lequel la surdité est totale et la rétinite évidente avant $10 \mathrm{ans}$, et un type II, plus tardif, avec une surdité incomplète et une fonction vestibulaire normale. Les deux types ne semblent pas alléliques : dans deux articles parus en $1990[6,7]$, le type II a pu être localisé dans la partie distale du bras long du chromosome 1 , alors que cette localisation pouvait être exclue pour le type 1. Le travail que viennent de publier Cremers et al. [2] conduit à considérer le gène $h C H M L$ comme un candidat au moins possible pour le type II du syndrome de Usher. A notre connaissance, cette hypothèse prometteuse n'a pas encore été vérifiée par l'analyse de ce gène chez des malades atteints de syndrome de Usher ; tout au moins, aucun résultat n'a pas encore été publié, mais on peut s'attendre à en entendre parler dans un délai très bref.

J.C.D.

1. Cremer FPM, van de Pol DJR, van Kerkhoff LPM, Wieringa B, Ropers $\mathrm{HH}$. Cloning of a gene that is rearranged in patients with choroïderemia. Nature 1990 ; 347 : 674-7.

2. Van den Hurk JAJM, van de Pol TJR, Molloy CM et al. Detection and characterization of point mutations in the choroideremia candidate gene by PCR-SSCP analysis and direct DNA sequencing. $A m$ I Hum Genet 1992 ; 50 : 1195-202.

3. Cremers FPM, Molloy CM, van de Pol DJR et al. An autosomal homologue of the choroïderemia gene colocalizes with the Usher syndrome type II locus on the distal part of chromosome 1q. Hum Molec Genet $1992 ; 1: 71-5$.

4. Fodoer E, Lee RT, O'Donnell JJ. Analysis of choroïderemia gene. Nature $1991 ; 351: 614$.

5. Bowes $\mathrm{C}, \mathrm{Li} \mathrm{T}$, Danciger $\mathrm{M}$, Baxter LC, Applebury ML, Farber DB. Retinal degeneration in the $r d$ mouse is caused by a defect in the $\beta$ subunit of rod cGMPphosphodiesterase. Nature 1990 ; 347 : 677-9.

6. Kimberling WJ, Weston MD, Möller C et al. Localization of Usher syndrome type II to chromosome 1q. Genomics 1990 ; 7 : 245-9.

7. Lewis RA, Otterud B, Stauffer D, Lalouel JM, Leppert M. Mapping recessive ophtalmic diseases : linkage of the locus for Usher syndrome type I : to a DNA marker on chromosome 1q. Genomics 1990 ; $7: 250-6$. 